\title{
Application of Adaptive Chaotic Simulated Annealing Network to Uplink Power Control in Asynchronous CDMA System
}

\author{
$\mathrm{Di} \mathrm{He} \mathrm{e}^{1,2 *}$ \\ ${ }^{1}$ Shanghai Key Laboratory of Navigation and Location-based Services, Shanghai Jiao Tong University, Shanghai, P.R. China \\ ${ }^{2}$ National Mobile Communications Research Laboratory, Southeast University, Nanjing, P.R. China \\ ${ }^{*}$ Corresponding author: Di He, Shanghai Key Laboratory of Navigation and Location-based Services, Shanghai Jiao Tong University, Shanghai, P.R. \\ China, Tel: +86-21-3420-4635; E-mail: dihe@sjtu.edu.cn
}

Received Date: November 09, 2017; Accepted Date: November 23, 2017; Published Date: November 25, 2017

Copyright: (C2017 He D. This is an open-access article distributed under the terms of the Creative Commons Attribution License; which permits unrestricted use; distribution; and reproduction in any medium; provided the original author and source are credited.

Citation: He D (2017) Application of Adaptive Chaotic Simulated Annealing Network to Uplink Power Control in Asynchronous CDMA System. Br J Res Vol.4 No 5: 32.

\section{Abstract}

A kind of adaptive Chaotic Stimulated Annealing (CSA) network approach used in the uplink power control of asynchronous Code Division Multiple Access (CDMA) mobile communication system is proposed in this study. The particular influence of near-far effect can be overcome by utilizing the global optimal searching function of CSA method, and the Multiple Access Interference (MAI) in certain cell can be conquered by introducing adaptive multiuser interference cancellation network in corresponding close loop control strategy. Computer simulations give the comparison results between proposed approach and conventional method with heterogeneous Signal-toInterference Ratio (SIR) threshold decision. It shows that this new approach can achieve higher channel capability and lower average outrage probability with some small increase of iteration steps, which can improve the performance of CDMA mobile communication system effectively.

Keywords: Code division multiple access (CDMA); Power control; Chaotic simulated annealing (CSA); Multiple access interference (MAI); Signal-to-interference ratio (SIR)

\section{Introduction}

In the code division multiple access (CDMA) mobile communication system, near-far effect is the most important factor that induces the unbalanced power received by the base station from different users within a cell. In other words, if the transmission power from each user is the same, the receiving power will be reduced along with the increasing of distance between base station and certain mobile terminal. When the power of certain user is too big, its interference to other users in the same cell will be enhanced. Otherwise, when the power is too small, the signal-to-interference ratio (SIR) will be reduced, which may result in the rising of bit error rate (BER) and the descending of mobile communication quality. Therefore, nearfar effect is a fatal problem that should be solved [1-3].
Power control is the most expedient means to overcome the near-far effect. It includes two aspects as uplink power controls and downlink power controls, respectively. Under uplink circumstances, open loop and close loop power control strategy is frequently used to adjust the different gain of each user to compensate the attenuation in the wireless channel [4], where open loop strategy needs less time during the adjustment process but close loop strategy can get higher precision of detection. In recent years, uplink power control algorithm with adaptive SIR thresholds [5] and optimum power control with heterogeneous thresholds [6] are widely discussed. Although these methods operate successfully under wireless communication circumstances, the problem of multiple access interference (MAl) and the speed of control required could not be solved successfully. Thus the real application scope of these methods is also bounded in this way.

In recent years, a kind of optimization method based on Stimulated Annealing (SA) has been proposed and applied in different areas. The SA methodology is a stochastic optimization algorithm based on Monte-Carlo iterative solution strategy. Its starting point is based on the similarity between the annealing process of solid material in physics and the general combinatorial optimization problem. The simulated annealing algorithm starts with a higher initial temperature and decreases with the decrease of temperature parameters. The global optimal solution of the objective function is found randomly in the solution space according to the probability jump property, that is, the local optimal solution can jump out of the probability and finally tend to the global optimum. Now this kind of methodology has been widely used in buffer allocation [7], water networks management [8], motion capture system [9], and manufacturing [10] and so on.

In this research, we propose a new approach to solve the problem of uplink power control. It is based on the combination of chaotic simulated annealing (CSA) and adaptive neural network. It can achieve higher channel capability and reduce the average transmitting power of mobile terminals than those with heterogeneous SIR thresholds. The computer simulation results also show the effectiveness of proposed method. 
The main contributions and novelties of our research work can be summarized as follows: (1) A kind of novel and dynamic uplink power control method used in the asynchronous CDMA system is proposed; (2) The detailed structure of the proposed adaptive chaotic simulated annealing network is given, which may be helpful for the real applications; (3) The SIR and BER performances of the proposed approach are analyzed, which are also the basis for the performance comparison with some other conventional power control methods; (4) The corresponding simulated BER performance, the average channel capability performance, the average outrage probability performance together with the iteration step number performance are carried out with comparisons with the conventional methods, which show the validity and applicability of the proposed approach.

The remains of the paper are arranged as follows: Section 2 gives the basic introduction to uplink power control in asynchronous CDMA system; Section 3 presents the idea of adaptive CSA power control method; and the comparison computer simulation results are given in Section 4; at last in Section 5 , some conclusions and summarizations are drawn to the whole paper.

\section{Uplink Power Control of Asynchronous CDMA System}

Asynchronous CDMA system is frequently discussed in the researching problem of uplink power control; because the burst of information signals to be transmitted by some certain user within a cell is unpredictable under practical environment. Assume the signal transmitted from certain user $k$ can be denoted by

$$
s_{k}(t)=c_{k}(t) \cdot d_{k}(t)
$$

Where, $c_{k}(t)$ is the address code of user $k$ with code period $T_{c}$, and $d_{k}(t)$ is the information code of user $k$ with symbol period $T_{\mathrm{b}}$.

Here we have $T_{\mathrm{b}}=N T_{\mathrm{c}}$, where $N$ is the bandwidth expansion factor in the asynchronous CDMA system.

Then the mixed signal received by some certain base station can be expressed by

$$
\begin{aligned}
& \phi(t)=\sum_{k=1}^{k}\left[A_{k} \cdot S_{k}\left(t-\tau_{k}\right)\right]+n(t), \\
& A_{k}=r_{k}^{-\rho_{k}} \cdot 10^{n / k^{10}}
\end{aligned}
$$

In Eq. (2), $K$ is supposed to be the total number of mobile users within the covering area of the base station; $A_{k}$ is the attenuation amplitude to the original signal $s_{\mathrm{k}}(t)$ of user $k$; is the time delay from transmitter to receiver; $n(t)$ is the additive white Gaussian noise (AWGN) in the wireless channel with mean 0 and variance. In Eq. (3), $r_{k}$ is the distance between the base station and user $k ; \rho_{\mathrm{k}}$ is the attenuation slope of transmitting path; and $\rho_{\mathrm{k}}$ is a variable in lognormal distribution with mean 0 and variance.
From the equations above we can conclude that: the signal transmitted from some certain user which is nearer to the base station has smaller attenuation than others, and it may cause serious MAl which can lead to unpredictable influences. The regular discussed method to solve such kind of near-far effect is the uplink power control $[1,4]$. By introducing the transmitting power gains to mobile users respectively, we want to keep the received power belonging to each user in the base station balanced. In the close loop power control, the base station should measure the attenuation of different users, and then feed corresponding gain adjustment information back to the users. After that, the signal in Eq. (2) can be changed to following form

$$
\phi(t)=\sum_{k=1}^{k}\left[A_{k} \cdot G_{k} \cdot S_{k}\left(t-\tau_{k}\right)\right]+n(t),
$$

where, $G_{\mathrm{k}}$ is the transmitting gain of user $k$.

Close loop power control is a dynamic process during the whole communication process, because the position of some certain user may be changing from time to time. So the measurement of base station and the gain adjustment of mobile user should both be dynamic. The methods of uplink power control proposed in $[5,6]$ are all based on heterogeneous SIR threshold, which can be denoted by

$$
\operatorname{SIR}_{i} \frac{A_{i} \cdot G_{i}}{\sum_{k=1}^{k}\left(A_{k} \cdot G_{k}\right)-A_{i} \cdot G_{i}+N_{0}}, \quad(i=1,2, \quad, K)
$$

where, $N_{0}$ is the power of AWGN in the wireless channel. All other users' signals are regarded as the interferences together with the channel noise. It needs to adjust $G_{\mathrm{i}}$ to ensure $S I R_{\mathrm{i}}$ bigger than some fixed threshold to fulfill the requirement of $B E R$.

By using the methods based on adaptive SIR thresholds or heterogeneous SIR thresholds, there exists one distinct disadvantage:

When the gain of $G_{\mathrm{i}}$ is increased, it can certainly guarantee the requirement of $S I R_{\mathrm{i}}$, but it may also enhance the MAI to other users, which leads to their descending of SIR.

\section{Proposed Method of Adaptive Chaotic Simulated Annealing Network}

To overcome the shortcoming stated above, here we propose a novel approach of uplink power control utilizing the adaptive chaotic simulated annealing network.

As is known, under the ideal circumstances without MAI and AWGN in the wireless channel, the attenuated uplink transmitting power Ak of user $k$ received by the base station can be detected from the receiving signal when the estimated time delay value $\hat{\tau}_{\kappa}$ equals to the real time delay value $\tau_{\kappa}$. Due to the good self-correlation characteristics of the address codes in the asynchronous CDMA system, the correlation results will reach a global peak value while $\hat{\tau}_{\kappa}=\tau_{\kappa}$. Or in other words, we can get the attenuated uplink transmitting power by finding a global 
maximum value as follows $A_{k}=\max _{0 \leq \tau^{\wedge} \leq T_{b}}\left[\phi(t) \cdot c_{k}\left(t-\hat{\tau}_{k}\right)\right],(k=1,2, \quad \ldots, K)$.

Many methods have good properties in solving the optimization problems, in which chaotic simulated annealing (CSA) $[11,12]$ is a promising one. It was first introduced and discussed by Chen LN and Aihara K. It uses the searching property in the fractal structure of chaotic neural network to realize the globally optimal or near-optimal solutions. Unlike the conventional methods in solving the combinational optimization problems, the CSA method can avoid the local-optimal solutions effectively. Now this method has been widely used in some nondeterministic polynomial hard (NP-hard) class problems, such as the Traveling Salesman Problem (TSP) and a maintenance scheduling problem for generators in a practical power system.

In the conventional CSA model, an energy function should be defined, which can also be regarded as the objective function. Then a CSA model can be established as follows

$$
\left\{\begin{array}{l}
x_{i}(t)=\frac{1}{1+e^{-y_{i}(t) / \varepsilon}} \\
y_{i}(t+1)=h \cdot y_{i}(t)+\alpha \cdot\left(-\frac{\partial E}{\partial x_{i}}\right)-z_{i}(t) \cdot\left[x_{i}(t)-I_{0}\right] \\
(i=1,2, \ldots), \\
z_{i}(t+1)=(1-\beta) \cdot z_{i}(t)
\end{array}\right.
$$

where, $\varepsilon$ is the steepness parameter of the output function; $h$ is the damping factor of nerve membrane; $\alpha$ is the positive scaling parameter for the inputs; $E$ is the energy function to be detected; $I_{0}$ is a fixed positive parameter; $B$ is the damping factor of the time-dependent $z_{\mathrm{i}}(t)$.

As in the optimization problems, an objective function should always be defined to get the optimal performance of the problem discussed. And when the objective function reaches its maximal or minimal value, the optimization problem can be solved. Here in the conventional CSA model, the objective function is always described as a kind of energy function which corresponds to the entropy of the system and can be generally defined as

$$
E=\min _{\text {conditions }}\left[\text { Entropy }\left(\mathrm{x}_{\mathrm{i}}, \mathrm{y}_{\mathrm{i}}, \mathrm{z}_{\mathrm{i}}\right)\right]
$$

where, $E$ is the energy function, and Entropy $\left(x_{i}, y_{i}, z_{i}\right)$ is the entropy of the system.

To solve the problem of uplink power control by using the CSA method, we can set the correlation output result at the base station as the energy function in Eq. (8). That is to say that to some certain user $k$, the relationship between $A_{k}$ in Eq. (6) and its energy function is

$$
\begin{aligned}
& E_{k}=-A_{k}=\min _{0 \leq \hat{\tau} \leq T_{b}}\left[\phi(t) \cdot c_{k}\left(t-\hat{\tau}_{k}\right)\right], \\
& (k=1,2, \ldots, K)
\end{aligned}
$$

As is known, $\phi(t)$ is a signal consists of multiple users information, where MAl is unavoidable. In addition, based on the near-far effect mentioned in Section 2, sometimes the MAI will cause serious influences to the prediction of attenuation power of certain user. But in the conventional correlation detection, MAl is not taken into the consideration. So to enhance the efficiency of power estimation, we reset the energy function of each user as $E_{i}=\min _{0 \leq \hat{\tau}_{i} \leq T_{b}}\left\{\left[\phi(t)-\sum_{\substack{k=1 \\ k \neq 1}}^{\mathrm{K}} c_{k}\left(t-\hat{\tau}_{\kappa}\right)\right] \cdot c_{i}\left(t-\hat{\tau}_{i}\right)\right\}$,

$(k=1,2 \ldots, K)$

From Eq. (10) it can be found that, when the time delay of each user within a cell can be estimated successfully, the MA will be eliminated and the attenuation power can be estimated.

Under the transformation above, we can change the optimization estimation process to the attenuation power of each user in the base station by

$$
\begin{aligned}
& \left\{\begin{array}{l}
x_{k, i}(t)=\frac{1}{1+e^{-y_{k, i}(t) / \varepsilon}} \\
y_{k, i}(t+1)=h \cdot y_{k, i}(t)+\alpha \cdot\left(-\frac{\partial E_{k}}{\partial x_{k, i}}\right)- \\
z_{k, i}(t) \cdot\left[x_{k, i}(t)-I_{0}\right] \\
z_{k, i}(t+1)=(1-\beta) \cdot z_{k, i}(t)
\end{array}\right. \\
& (k=1,2, \ldots, K ; i=1,2, \ldots) .
\end{aligned}
$$

In the modified CSA model, the optimization process of different user is not independent to each other at all, because the energy function $E_{\mathrm{k}}$ has certain relationship with all users in the cell. In other words, the whole optimization process is a dynamic adaptive process. During the searching period, the trace of CSA is not only influenced by its own dynamic evolvement, but also influenced by the optimization trace of other users. So the interaction of each user makes the uplink power control may be realized by an all connected network, and each is composed of the standard units with 3 cell $x_{k, i}(t), y_{k, i}(t)$ and $z_{k, i}(t)$. When the transient chaotic phenomenon in each unit disappears, the optimization results will be the attenuation power in the uplink path with

$$
\begin{aligned}
& A_{k}=-E_{k}=-\min _{0 \leq \hat{\tau}_{i} \leq T_{b}}\left[\phi(t) \cdot c_{k}\left(t-\hat{\tau}_{k}\right)\right], \\
& (k=1,2, \ldots, K)
\end{aligned}
$$

Then, the base station can distribute different channel transmitting gain to each user according to their differences in the channel attenuation. To diminish the total receiving power in the base station, set the maximum receiving power among different users as the standard of adjustment, then we can get

$$
G_{k}=\frac{\max _{k=1,2, \ldots, K} A_{k}}{A_{k}}, \quad(k=1,2, \ldots, K)
$$

When the receiving power of each user is balanced after the power control, the channel capability can certainly be improved, and the BER properties may also be improved effectively. While the corresponding BER can be measured by 
$B E R_{k}=Q\left(S I R_{k}\right)$

$(k=1,2, \ldots, K)$

where, $S I R_{\mathrm{k}}$ is the SIR of user $k ; Q(\cdot)$ is the standard normaldistribution probability integral function. And the channel capability can be obtained by

$$
\frac{C}{B}=\log _{2}(1+\overline{S I R})^{\wedge}=\log _{2}\left(1+\frac{1}{K} \cdot \sum_{k=1}^{K} S I R_{k}\right)
$$

where, $\overline{S I R}$ is the average value of SIR of all users within a cell.

To give a clear presentation to the proposed method in uplink power control optimization process, the structure of the proposed adaptive CSA network is plotted in Figure 1. It can be found that this structure can be realized by some basic electronic circuits conveniently.

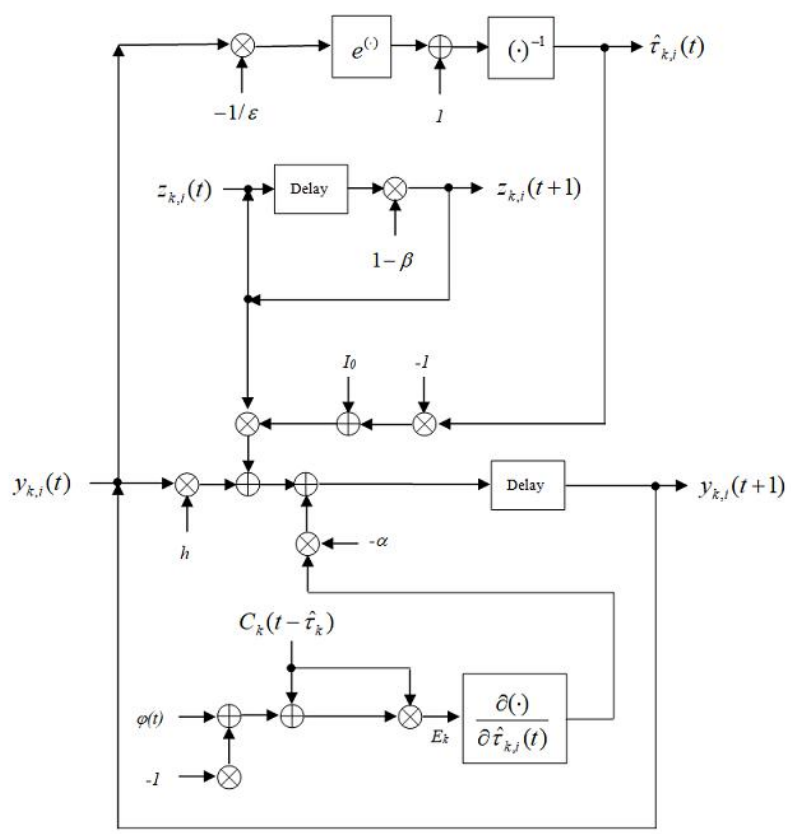

Figure1. Structure of Adaptive Chaotic Simulated Annealing Network.

\section{Computer Simulation Results}

To compare the performances of the proposed adaptive CSA network with those of the method using heterogeneous SIR threshold decision method in [6], we give out some computer simulation results in this section. In the computer simulations, $m$ sequences with length 127 are selected as the CDMA address codes. The total number of mobile users within the cell is $K=15$ when this parameter is fixed. Especially in the adaptive CSA process, the parameters in Eq. (10) are set as: $\varepsilon=0.004, h=0.9$, $\alpha=0.001,10=0.65,6=0.001$.

Figure 2 shows the BER performance comparison results of different methods. While in plotting the theoretical results in the simulations, the Distributed Power Control Algorithm introduced in [6] is used to give the theoretical performance. The circumstances without power control and ideal power control are also introduced to give the ultimate conditions of uplink power control. For the theoretical results without power control, it is assumed that the MAI in calculating $S I R_{\mathrm{i}}$ in Eq. (5) will remain the same as it received at the receiver without any MAI cancellation algorithm. And for the theoretical results under ideal power control, it is assumed that the MAI in calculating $S I R_{\mathrm{i}}$ in Eq. (5) will reach its minimal value by using some optimal MAI cancellation algorithm. In other words, the circumstances without power control gives the worst results, on the contrary, circumstances under ideal power control gives the best results. From the figure it can be discovered clearly that proposed CSA network power control method has better BER performance than the method with heterogeneous SIR threshold decision [6].

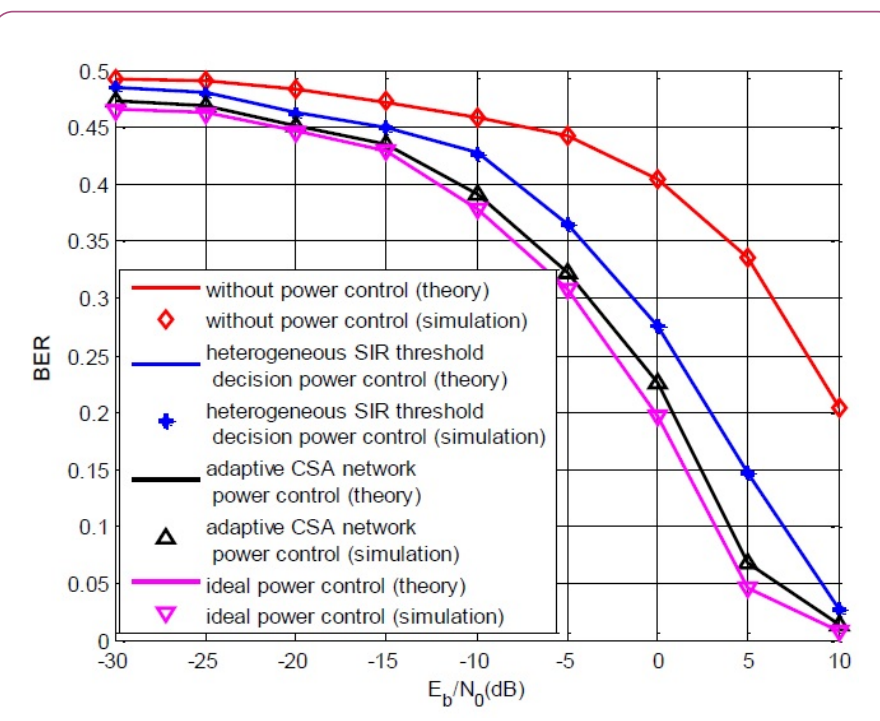

Figure 2. BER performance under different uplink power control methods.

Figure 3 shows the average channel capabilities of different methods under various signal-to-noise ratios (SNR) circumstances. And the methods to get the theoretical results remain the same as described in plotting Figure 2 . From the figure it can be observed that the proposed adaptive CSA network power control method can improve the channel capability effectively compared with the method with heterogeneous SIR threshold decision [6], and its performance is much closer to that of the ideal power control circumstance, which certifies the validity of the proposed method. At the same time, it can be found that the theoretical results and the computer simulation results in all methods coincide well, which also guarantee the analyses correctness of the proposed approach.

Simultaneously, Figure $\mathbf{4}$ gives the average outrage probability performances under different uplink power control methods, which include those without power control, the SIR threshold decision power control, the heterogeneous SIR threshold decision power control [6], and the proposed adaptive CSA network power control. The computer simulation parameters are selected as the same used in the simulations in Figure $\mathbf{2}$ and Figure 3. While it can be found in the results that the capacities at the outage probability of $1 \%$ achieved by the above 
algorithms are 14, 21, 30 and 31 mobiles/cell, respectively. And by using the proposed adaptive CSA network power control method, the capacity gains over the other methods can be observed clearly. Finally, to evaluate the convergence speed and the delay of the proposed approach, Figure 5 plots the iteration numbers of different uplink power control methods between the received SIR and the required SIR. In this computer simulation, the active mobile density is $\mathbf{2 9}$ mobiles/cell, and the balanced ratio is set as 1.0012 . It can be discovered that the maximum and minimum of ratios among all the mobiles converge to the balanced ratio are about 18 and 20 iterations by using the SIR threshold decision power control and the heterogeneous SIR threshold decision power control [6], respectively, and that by using the proposed adaptive CSA network power control method is about 22 iterations. So it can be concluded that although it may need some more iteration steps for the proposed approach compared with the SIR threshold decision power control method in [6], the increasing number of the iterations is very small which can be ignored to some extent, while the BER performance, the average channel capability performance and the average outrage probability performance can all be improved, which is certainly worthy of the small increasing of the iteration steps needed.

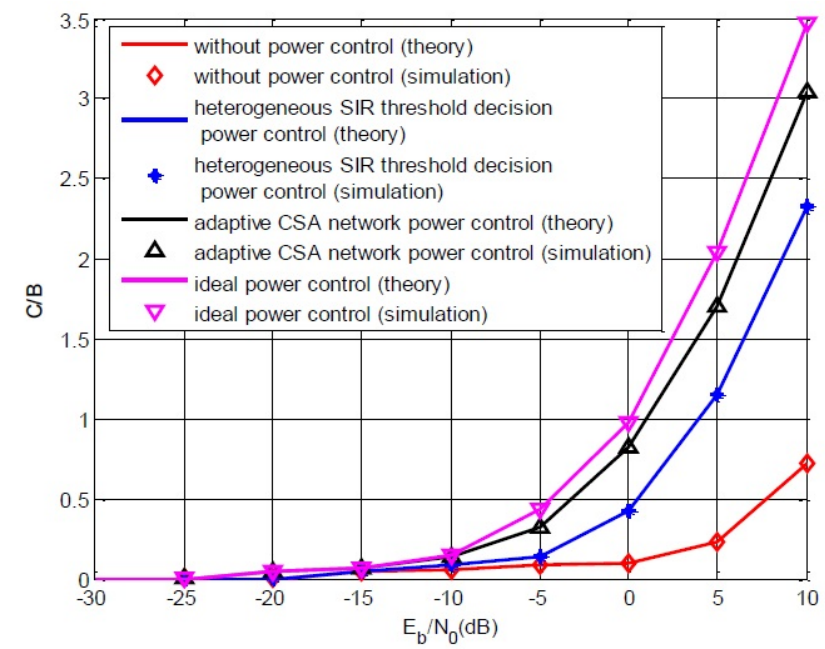

Figure 3. Average channel capabilities under different uplink power control methods.

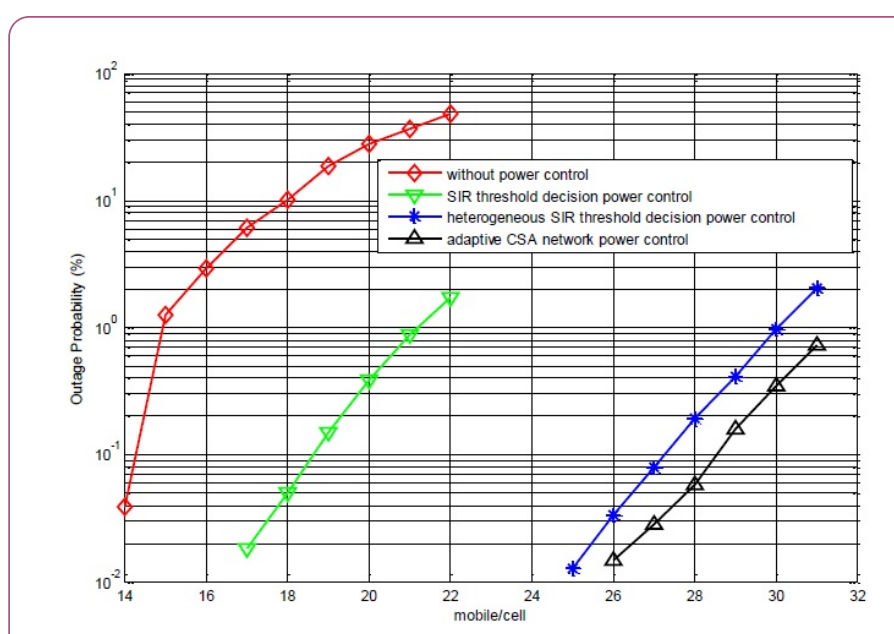

Figure 4. Average outrage probability performances under different uplink power control methods.

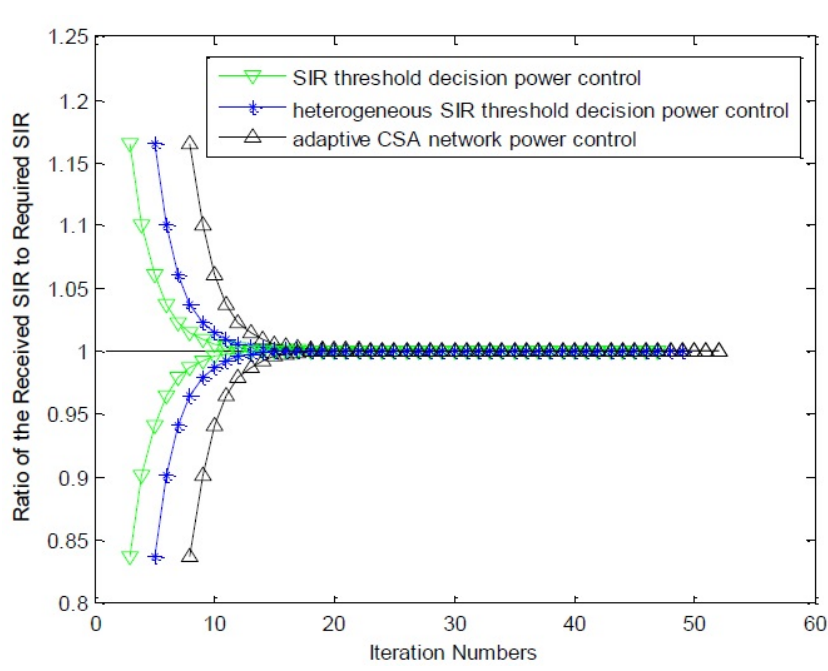

Figure 5. Iteration numbers of different uplink power control methods between the received SIR and the required SIR.

\section{Conclusion}

In this paper, a new method of adaptive CSA network is proposed to solve the key problem of uplink power control in CDMA mobile communications systems. A novel structure of the proposed adaptive CSA network is presented, while the SIR and BER performances are also given to show the advantages of the proposed methodology. By introducing the scheme of MAI cancellation, the optimization processes of channel attenuation prediction to each user are becoming interactive. When the annealing process of different users finishes at last, the steady network structure will give out the transmitting gain successfully. In the computer simulations, the BER performance, the average channel capability, the average outrage probability performance, and the iteration numbers show the advantages of this proposed method over the conventional method with heterogeneous SIR threshold decision. It will certainly improve the performance of the receiving systems effectively. 


\section{Acknowledgement}

This research work is supported by the National Natural Science Foundation of China under Grant Nos. 61771308 and 91438113, the Important National Science and Technology Specific Project of China under Grant No. 2016ZX03001022-006, the Shanghai Science and Technology Committee under Grant No. 16DZ1100402, and the open research fund of National Mobile Communications Research Laboratory of Southeast University of China under Grant No. 2017D11.

\section{References}

1. Diamant R, Casari P, Campagnaro F, Zorzi M (2016) A HandshakeBased Protocol Exploiting the Near-Far Effect in Underwater Acoustic Networks. IEEE Wireless Communications Letters 5:308-311.

2. Huang P, Pi Y (2011) Urban Environment Solutions to GPS Signal Near-Far Effect. IEEE Aerospace and Electronic Systems Magazine 26: 18-21.

3. Kabir P, Shafinia MH, Marvasti F (2013) Capacity Bounds of Finite Dimensional CDMA Systems with Power Allocation, Fading, and Near-Far Effects. IEEE Communications Letters 17: 15-18.

4. Viterbi AJ (1995) CDMA: principles of spread spectrum communication. Addison-WeSley Publishing Company.

5. Firdaus, Muayyadi A, Astuti RP (2012) Performance analysis of adaptive power control based on Signal to Interference Ratio (SIR) using fuzzy genetic for WCDMA. 2012 Second International Conference on Digital Information and Communication Technology and it's Applications (DICTAP'2012), 354-358.

6. Qiang Wu (2000) Optimum Transmitter Power Control in Cellular Systems with Heterogeneous SIR Thresholds. IEEE Transactions on Vehicular Technology 49: 1424-1429.

7. Spinellis DD, Papadopoulos CT (2000) A Simulated Annealing Approach for Buffer Allocation in Reliable Production Lines. Annals of Operations Research 93: 373-384.

8. Pérez-Sánchez $M$, Sánchez-Romero FJ, López-Jiménez PA, Ramos HM (2017) PATs Selection Towards Sustainability in Irrigation Networks: Simulated Annealing as a Water Management Tool. Renewable Energy 116: 234-249.

9. Matthias L, Bruno D (2006) A Markerless Motion Capture System to Study Musculoskeletal Biomechanics: Visual Hull and Simulated Annealing Approach. Annals Biomedical Engineering 34: 1019.

10. Raaymakersa WHM (2000) Scheduling Multipurpose Batch Process Industries with No-wait Restrictions by Simulated Annealing. European Journal of Operational Research 126: 131-151.

11. Chen S (2011) Chaotic Simulated Annealing by a Neural Network With a Variable Delay: Design and Application. IEEE Transactions on Neural Networks 22: 1557-1565.

12. Wang L, Li S, Tian F, Fu X (2004) A Noisy Chaotic Neural Network for Solving Combinatorial Optimization Problems: Stochastic Chaotic Simulated Annealing. IEEE Transactions on Systems, Man, and Cybernetics, Part B (Cybernetics) 34: 2119-2125. 